\title{
NUCLEAR SUBALGEBRAS OF UHF C*-ALGEBRAS
}

\author{
by R. J. ARCHBOLD and ALEXANDER KUMJIAN
}

(Received 6th February 1985)

\section{Introduction}

A $C^{*}$-algebra $A$ is said to be approximately finite dimensional $(A F)$ if it is the inductive limit of a sequence of finite dimensional $C^{*}$-algebras (see [2], [5]). It is said to be nuclear if, for each $\mathrm{C}^{*}$-algebra $B$, there is a unique $\mathrm{C}^{*}$-norm on the ${ }^{*}$-algebraic tensor product $A \otimes B$ [11]. Since finite dimensional $C^{*}$-algebras are nuclear, and inductive limits of nuclear $\mathrm{C}^{*}$-algebras are nuclear [16], every $\mathrm{AF} \mathrm{C}^{*}$-algebra is nuclear. The family of nuclear $C^{*}$-algebras is a large and well-behaved class (see [12]). The AF $\mathrm{C}^{*}$-algebras form a particularly tractable sub-class which has been completely classified in terms of the invariant $K_{0}[7]$, [5]:

Choi [4] showed that a $C^{*}$-subalgebra of a nuclear $C^{*}$-algebra can fail to be nuclear. Since the larger algebra in Choi's example is not AF, there remained the question as to whether a $C^{*}$-subalgebra of an AF $C^{*}$-algebra is ncessarily nuclear (see [6, Problem 10] and [12, Problem 3]). Blackadar [1] has recently answered this question negatively by showing that every non-type $\mathrm{I} \mathrm{C}^{*}$-algebra [13] contains a non-nuclear $\mathrm{C}^{*}$-subalgebra. Nevertheless, in special circumstances it is possible to give a positive answer. In Theorem 3 we show that if $D$ is a $C^{*}$-subalgebra of a uniformly hyperfinite $C^{*}$-algebra $A$ (see below) and if $D$ contains a suitable maximal abelian self-adjoint subalgebra (masa) of $A$ then $D$ is not only nuclear but even AF. In Remark 4 we indicate how this result may be generalized to the case where $A$ is a unital AF $C^{*}$-algebra.

\section{Preliminaries}

A $\mathrm{C}^{*}$-algebra $A$ is said to be uniformly hyperfinite $(U H F)$ if it is the norm-closure of the union of an ascending sequence of subalgebras each of which is *-isomorphic to a full complex matrix algebra and contains the identity of $A$ [9]. Thus any UHF $\mathrm{C}^{*}$-algebra is automatically AF. If $A$ is a UHF $\mathrm{C}^{*}$-algebra then it is *-isomorphic to an infinite tensor product $\bigotimes_{i \geqq 1} A_{i}$ where $A_{i}=M_{n(i)}$ (the $\mathrm{C}^{*}$-algebra of all $n(i) \times n(i)$ complex matrices) for some positive integer $n(i)$ [14, Section 4]. A masa $B$ of a UHF $\mathrm{C}^{*}$-algebra $A$ is said to be a standard diagonal if there exists a *-isomorphism $\theta$ of $A$ onto $\bigotimes_{i \geqq 1} A_{i}$, where each $A_{i}=M_{n(i)}$ for some $n(i)$, such that $\theta(B)=\bigotimes_{i \geqq 1} B_{i}$ where each $B_{i}$ is a masa of $A_{i}$ (see [10] for a more general notion). It is well-known that $\bigotimes_{i \geqq 1} B_{i}$ is indeed a masa of $\bigotimes_{i \geqq 1} A_{i}$ (see [17, Corollary 11] for a more general result).

Lemma 1. Let $A=\bigotimes_{i \geqq 1} A_{i}$, where each $A_{i}=M_{n(i)}$ for some $n(i)$, and let $B=\bigotimes_{i \geqq 1} B_{i}$ 
where each $B_{i}$ is a masa of $A_{i}$, so that $B_{i}=\mathbb{C} e_{1}^{(i)}+\cdots+\mathbb{C} e_{n(i)}^{(i)}$ for some maximal orthogonal family $\left\{e_{1}^{(i)}, \ldots, e_{n(i)}^{(i)}\right\}$ of minimal nonzero projections in $A_{i}$.

For each $i$, let $R_{i}$ be the projection of norm one from $A_{i}$ onto $B_{i}$ given by

$$
R_{i}(a)=\sum_{j=1}^{n(i)} e_{j}^{(i)} a e_{j}^{(i)} \quad\left(a \in A_{i}\right)
$$

Let $C_{i}=A_{1} \otimes \cdots \otimes A_{i} \otimes\left(\bigotimes_{j>i} B_{j}\right)$. For $n>i$ let $P_{i}^{(n)}=\mathrm{id}_{1} \otimes \cdots \otimes \cdots \otimes \mathrm{id}_{i} \otimes R_{i+1} \otimes \cdots$ $\otimes R_{n} \otimes \mathrm{id}$. Then there exists a projection of norm one $P_{i}=\mathrm{id}_{1} \otimes \cdots \otimes \mathrm{id}_{i} \otimes\left(\otimes_{j>i} R_{j}\right)$ of $A$ onto $C_{i}$ such that

(i) $P_{i}(x)=\lim P_{i}^{(n)}(x) \quad(x \in A)$,

(ii) $P_{i}=P_{i} P_{i+1}$ for each $i$,

(iii) for any $C^{*}$-algebra $C$ satisfying $B \subseteq C \subseteq A, P_{i}(C) \subseteq C$,

(iv) $x=\lim P_{i}(x) \quad(x \in A)$.

Proof. The construction of $P_{i}$ is routine so we merely sketch the argument. Let $a \in A$ and let $a=\lim a_{n}$ where $a_{n} \in A_{1} \otimes \cdots \otimes A_{n} \otimes \mathbb{C} 1$. For $m>n>i$,

$$
\left\|P_{i}^{(n)}\left(a_{n}\right)-P_{i}^{(m)}\left(a_{m}\right)\right\|=\left\|P_{i}^{(m)}\left(a_{n}\right)-P_{i}^{(m)}\left(a_{m}\right)\right\| \leqq\left\|a_{n}-a_{m}\right\| .
$$

So $\left(P_{i}^{(n)}\left(a_{n}\right)\right)_{n \geqq i}$ is a Cauchy sequence in $C_{i}$. The limit is independent of $\left(a_{n}\right)$ and is denoted by $P_{i}(a)$. It is straightforward to check that $P_{i}$ is a projection of norm one from $A$ onto $C_{i}$.

Since $P_{i}^{(n)}(a) \rightarrow P_{i}(a)$ whenever $a \in A_{1} \otimes \cdots \otimes A_{k} \otimes \mathbb{C} 1$ for some $k$, (i) follows from the fact that $\left\|P_{i}^{(n)}\right\|=1$ for all $n>i$.

Clearly $P_{i}=P_{i} P_{i+1}$ on each algebra $A_{1} \otimes \cdots \otimes A_{k} \otimes \mathbb{C} 1$, and so (ii) holds by continuity.

Let $c \in C$. Since $B \subseteq C$, it follows from the definition of the $R_{k}$ 's that $P_{i}^{(n)}(c) \in C$. Hence (iii) follows from (i).

Since $P_{i}(a) \rightarrow a$ whenever $a \in A_{1} \otimes \cdots \otimes A_{k} \otimes \mathbb{C} 1$ for some $k$, (iv) follows from the fact that $\left\|P_{i}\right\|=1$ for all $i$.

Proposition 2. Let $A=M_{n} \otimes B$ where $B$ is a unital abelian $A F \mathrm{C}^{*}$-algebra. Let $\left\{e_{1}, \ldots, e_{n}\right\}$ be a maximal orthogonal family of minimal nonzero projections in $M_{n}$. Suppose that $D$ is a $C^{*}$-subalgebra of $A$ such that $D \supseteq e_{i} \otimes B(1 \leqq i \leqq n)$. Then $D$ is an $A F C^{*}$ algebra.

Proof. For $1 \leqq i \leqq n$ let $J_{i}$ be the norm-closure of $D\left(e_{i} \otimes 1\right) D$, so that $J_{i}$ is a normclosed two sided ideal of $D$. Let

$$
D_{i}=\left(e_{i} \otimes 1\right) J_{i}\left(e_{i} \otimes 1\right)=\mathbb{C} e_{i} \otimes B
$$

Since $J_{i}\left(e_{i} \otimes 1\right) J_{i} \supseteq D\left(e_{i} \otimes 1\right) D, D_{i}$ is a full hereditary $C^{*}$-subalgebra of $J_{i}$ (see [3]). Since $J_{i}$ is separable, $J_{i} \otimes \mathscr{K} \cong D_{i} \otimes \mathscr{K}[3$, Theorem 2.8] (where $\mathscr{K}$ denotes the algebra of compact linear operators on a Hilbert space of countably infinite dimension). Thus 
$J_{i} \otimes \mathscr{K}$ is AF. If $e$ is a minimal nonzero projection in $\mathscr{K}$ then $J_{i} \cong(1 \otimes e)\left(J_{i} \otimes \mathscr{K}\right)(1 \otimes e)$ which is AF since it is a hereditary subalgebra of the AF algebra $J_{i} \otimes \mathscr{K}$ (see [8, Theorem 3.1], where a more general notion of "AF" is considered, and [5, Lemma 9.4]).

Since $\left(J_{1}+J_{2}\right) / J_{1} \cong J_{2} /\left(J_{1} \cap J_{2}\right),\left(J_{1}+J_{2}\right) / J_{1}$ is AF. It follows from the work of Brown and Elliott [5, Theorem 9.9] that $J_{1}+J_{2}$ is AF. Proceeding in the obvious way, one obtains that $J_{1}+J_{2}+\cdots+J_{n}=D$ is AF.

\section{The main result}

Theorem 3. Let $A$ be a UHF algebra and let $D$ be a $C^{*}$-subalgebra of $A$ which contains a standard diagonal $B$ of $A$. Then $D$ is an $A F C^{*}$-algebra (and hence is nuclear).

Proof. We may assume $B=\bigotimes_{i \geq 1} B_{i} \subseteq D \subseteq A=\bigotimes_{i \geq 1} A_{i}$ where $B_{i}$ is a masa of $A_{i}=M_{n(i)}$. Let $P_{i}(i \geqq 1)$ be as in Lemma 1. Let $D_{i}=P_{i}(D)$. By (iii),

$$
D \cap C_{i} \subseteq P_{i}(D) \subseteq D \cap C_{i} .
$$

Thus $D_{i}=D \cap C_{i}$, a (necessarily liminary) $\mathrm{C}^{*}$-subalgebra of the liminary $\mathrm{C}^{*}$-algebra $C_{i}$. Since $B \subseteq D \cap C_{i+1}=D_{i+1}$, it follows from (ii) and (iii) that $D_{i}=P_{i}\left(D_{i+1}\right) \subseteq D_{i+1}$. It follows from (iv) that $D=$ norm-closure $\left(\bigcup_{i \geq 1} D_{i}\right)$. Since $D_{i}$ is liminary and hence nuclear [16], the nuclearity of $D$ is now apparent. However we shall show that $D$ is actually AF.

By Proposition $2, D_{i}$ is AF ( $\left.i \geqq 1\right)$. Since $D$ is separable, it follows from [2, Theorem 2.2] that $D$ is $\mathrm{AF}$.

Remark 4. We indicate how the above result may be extended to the case where $A$ is a unital $\mathrm{AF} \mathrm{C}^{*}$-algebra. Let $\left\{A_{i}\right\}$ be an increasing sequence of unital finite dimensional subalgebras. To construct a standard diagonal in $A$, one chooses a sequence of masas $B_{i} \subseteq A_{i}$ inductively as in [15]; given $B_{i}$, let $B_{i+1}$ be generated by $B_{i}$ and a masa in the relative commutant of $A_{i}$ in $A_{i+1}$. Let $B$ denote the closure of $\cup B_{i}$ and note that $B$ is a hyperfinite diagonal in $A$ (see [10, Section 6.16]).

As in Lemma 1 above, there is for each $i$ a conditional expectational, $P_{i}: A \rightarrow C_{i}$ (where $C_{i}$ is the subalgebra generated by $B$ and $A_{i}$ ), satisfying properties (ii), (iii), (iv), (see [10, Proposition 6.15]). Hence, if $D$ is a subalgebra of $A$ which contains $B$, then it is generated by the family of subalgebras $\left\{D \cap C_{i}\right\}$. Since $D \cap C_{i}$ is an AF C*-algebra $\left(C_{i}\right.$ may be expressed as a finite direct sum of $C^{*}$-algebras of the form considered in Proposition 2), $D$ is an $\mathrm{AF} \mathrm{C}^{*}$-algebra, as well.

Acknowledgements. The first-named author is grateful to the University of California for supporting a visit to the Mathematical Sciences Research Institute at Berkeley during which this work was completed. The second author wishes to acknowledge the support of the Australian Research Grants Scheme and the hospitality of the M.S.R.I. 


\section{REFERENCES}

1. B. Blackadar, Nonnuclear subalgebras of $\mathrm{C}^{*}$-algebras, J. Operator Theory. (to appear).

2. O. Brattel, Inductive limits of finite dimensional $\mathrm{C}^{*}$-algebras, Trans. Amer. Math. Soc. 171 (1972), 195-234.

3. L. Brown, Stable isomorphism of hereditary subalgebras of $\mathrm{C}^{*}$-algebras, Pacific J. Math. 71 (1977), 335-348.

4. M-D. CHо, A simple $\mathrm{C}^{*}$-algebra generated by two finite-order unitaries, Canad. J. Math. 31 (1979), 867-880.

5. E. G. Efrros, Dimensions and $C^{*}$-algebras (CBMS Regional Conference Series No. 46, Amer. Math. Soc., Providence, R.I., 1981).

6. E. G. Efrros, On the structure of $C^{*}$-algebras: Some old and new problems, Proc. Symp. Pure Math. 38 (1982), 19-34.

7. G. A. Elliotr, On the classification of inductive limits of sequences of semi-simple finite dimensional algebras, J. Algebra 38 (1976), 29-44.

8. G. A. Elliotr, Automorphisms determined by multipliers on ideals of a $\mathrm{C}^{*}$-algebra, $J$. Functional Analysis 23 (1976), 1-10. 340.

9. J. Glimm, On a certain class of operator algebras, Trans. Amer. Math. Soc. 95 (1960), 318

10. A. Kumjian, On $C^{*}$-diagonals, preprint.

11. E. C. LANCE, On nuclear C*-algebras, J. Functional Analysis 12 (1973), 157-176.

12. E. C. LanCE, Tensor products and nuclear C*-algebras, Proc. Symp. Pure Math. 38 (1982), 379-399.

13. G. K. Pedersen, C*-algebras and their automorphism groups (Academic Press, London, 1979).

14. R. T. Powers, Representations of uniformly hyperfinite algebras and their associated Von Neumann rings, Annals of Math. 86 (1967), 138-171.

15. S. StRATILA and D. VoICUlesCu, Representations of AF algebras and of the group $U(\infty)$ (Lecture notes in Math. 486, Springer-Verlag, Berlin, 1975).

16. M. TAKESAK1, On the cross-norm of the direct product of $\mathrm{C}^{*}$-algebras, Tôhoku Math. J. 16 (1964), 111-122.

17. A. S. Wassermann, The slice map problem for $C^{*}$-algebras, Proc. London Math. Soc. (3) 32 (1976), 537-559.

\section{Department of Mathematics \\ UNIVERSITY OF CALIFORNIA \\ Santa Barbara \\ California, 93106 \\ USA}

Permanent Address:

Department of Mathematics

The Edward Wright BuILding

Dunbar Street

AberdeEn AB9 2TY

UK
School of Mathematics

UNIVERSITY OF New South Wales

KENSINGTON

N.S.W. 2033

Australia 